Tesis Doctoral:

\title{
"LA DIMENSIÓN ESPACIAL DE LA INSEGURIDAD URBANA EN EL SIGLO XXI. PERCEPCIÓN DEL RIESGO Y CONSTRUCCIÓN SOCIAL DEL MIEDO, EN LA CIUDAD DE RESISTENCIA. REPÚBLICA ARGENTINA".'
}

Oscar Luis Pyszczek

Directora: Dra. Cristina Valenzuela (UNNE)

C-Directora: Dra. María Eugenia Cepparo (UNCuyo)

\section{"La construcción social de los espacios de (in)seguridad ciudadana."}

\begin{abstract}
"El delito y la preocupación por la inseguridad conocen un curso ascendente en las últimas dos décadas. Se ha instalado como un problema público central, se ha transformado en un tema de conversación habitual, ha pasado de su lugar tradicional en los medios populares, a las páginas centrales de los considerados más prestigiosos, ha sido fuente de una constante demanda hacia el Estado y dinamizado un constante mercado."(Bergman
\end{abstract}

y Kessler, 2006).

\section{Situación.}

La (In)seguridad Delictiva Urbana, se ha transformado en una de las cuestiones más acuciantes de la vida moderna y al parecer, no conoce fronteras ni soluciones definitivas en los albores del nuevo siglo.

La complejidad del abordaje científico del fenómeno, radica en la multiplicidad de aspectos que integran su naturaleza: desde el punto de vista conceptual, es posible entenderlo y enfocarlo como un fenómeno social emergente (atendiendo a sus causas), como una problemática social (atendiendo principalmente a sus consecuencias) o bien, como una cuestión de debate que surge según los vaivenes de

\footnotetext{
${ }^{1}$ Defendida en la Universidad Nacional de Cuyo. (UNCuyo). Doctorado en Geografía.
}

Publicado en formato digital: Dr. Oscar Luis Pyszczek. "LA DIMENSIÓN ESPACIAL DE LA INSEGURIDAD URBANA EN EL SIGLO XXI. PERCEPCIÓN DEL RIESGO Y CONSTRUCCIÓN SOCIAL DEL MIEDO, EN LA CIUDAD DE RESISTENCIA. REPÚBLICA ARGENTINA". RESÚMENES DE TESIS. Revista Geográfica Digital. IGUNNE. Facultad de Humanidades. UNNE. Año 12. № 23. Enero - Junio. 2015. ISSN 1668-5180 Resistencia, Chaco.

En: http://hum.unne.edu.ar/revistas/geoweb/default.htm 
la política y los calendarios electorales (aspecto coyuntural). Desde el punto de vista metodológico conforma un desafío doblemente complejo que oscila entre la objetividad y la subjetividad de su naturaleza. Las divergencias al momento de determinar lo seguro o lo (In)seguro, y la consideración de ello estará empapado de las experiencias biográficas, contextos ideológicos, edad, sexo, etc.

En este sentido, la (in)seguridad delictiva urbana deviene en un universo de elementos, factores, procesos, causas y consecuencias, vinculados tan estrechamente, que conforman un campo de estudio casi ilimitado y un amplio espectro para los enfoques transdiciplinarios de la ciencia. Su naturaleza amplia tiene asidero genético desde su fuente: la "inseguridad delictiva", que es una denominación específica que resulta del desmembramiento de un concepto macro que la comprende: el de la "(in)seguridad" general.

Son varios los autores (Beck 2006, Curbet 2008, Zaluar, 2008 entre otros) que señalan la multiplicación de las ansiedades e incertidumbres en los tiempos actualesentre ellos, el sociólogo polaco ZygmuntBauman, quien define a los tiempos modernos como la era del "miedo líquido". Este interesante concepto alude a la pérdida de lo "sólido", de las certezas, de lo seguro, en manos de la incertidumbre que caracteriza los tiempos contemporáneos, a la ignorancia sobre la amenaza concreta que se cierne sobre el hombre y a la incapacidad de determinar que se puede hacer para contrarrestarla. Este hecho resulta paradójico puesto que, en palabras del mismo Bauman:

"La modernidad iba a ser aquel período de la historia humana en el que, por fin, nos sería posible dejar atrás los temores que dominaron la vida social del pasado, hacernos con el control de nuestras vidas $y$ dominar las descontroladas fuerzas del mundo social y natural."(2006: 17).

Ante estas circunstancias, ¿Por qué razón no se pudo contener y controlar las incertidumbres del presente?, ¿Cuál/es es/son la/s causa/s que produjo la multiplicación de los miedos, temores e incertidumbres en la vida moderna? La respuesta no resulta sencilla y merece una extensa revisión ontológica, que excede holgadamente cualquier intento. No obstante, y a modo general, podemos mencionar

Publicado en formato digital: Dr. Oscar Luis Pyszczek. "LA DIMENSIÓN ESPACIAL DE LA INSEGURIDAD URBANA EN EL SIGLO XXI. PERCEPCIÓN DEL RIESGO Y CONSTRUCCIÓN SOCIAL DEL MIEDO, EN LA CIUDAD DE RESISTENCIA. REPÚBLICA ARGENTINA". RESÚMENES DE TESIS. Revista Geográfica Digital. IGUNNE. Facultad de Humanidades. UNNE. Año 12. № 23. Enero - Junio. 2015. ISSN 1668-5180 Resistencia, Chaco.

En: http://hum.unne.edu.ar/revistas/geoweb/default.htm 
que la globalización actual supuso cambios estructurales en tres ejes principales: lo que respecta a lo relacional-comunitario, a lo político y a lo económico. En cada una estas esferas los individuos que conforman la sociedad, encuentran la contención para sentirse "integrados". Pero por el contrario, la actual precariedad económica, la individualización, la desafiliación política y el insuficiente reconocimiento de derechos sociales son algunas de sus principales expresiones, generadoras de nuevas realidades de (in)seguridad social. Este aspecto de los tiempos contemporáneos, se presenta en una "simultaneidad escalar", es decir, actúa a nivel planetario-mundial, con repercusiones regional-local, por lo tanto son pocos los sitios del mundo que escapan a sus consecuencias y a la proliferación de las ansiedades e incertidumbres modernas.

Vivimos en tiempos donde los peligros y los miedos se multiplican y no es posible establecer un asidero o foco permanente. La (in)seguridad reside a toda hora y en todo lugar. En muchos casos resulta complejo desmembrar las (in)seguridades, puesto que no se presentan de forma individualizada sino combinadas: de allí el origen de la clásica distinción entre los miedos concretos (donde se identifica una causa específica) y los difusos (a veces denominados "sensación de (in)seguridad").

Pero, ¿Qué es la (in)seguridad? Específicamente el término "(in)seguridad", puede ser entendido en múltiples acepciones y formas. Según la Real Academia Española, la inseguridad es la falta o ausencia deseguridad, término que deriva del latín securitas refiriendo a aquello que está "exento de peligro, daño o riesgo, o que es cierto, firme e indubitable". ${ }^{2}$ De este modo su concepto puede designar diferentes contextos y diversas circunstancias, pero en todas ellas subyace el componente de riesgo, peligroo bienincertidumbre por "algo" que atente contra nuestras vidas, bienes o seres queridos o que pueda llegar a ocurrir. En el caso particular del tema que nos convoca, la fuente de incertidumbre, de (in)seguridad estará dada por la actividad delictiva con asidero en la ciudad de Resistencia.

Es menester mencionar que debido a que la existencia y utilización del vocablo (In)seguridad se debe a la carencia o falta de "seguridad", se ha considerado atinado mencionar a lo largo de la tesis la sigla (In)- entre paréntesis- aludiendo precisamente

${ }^{2}$ Disponible en: http://definicion.de/inseguridad.

Publicado en formato digital: Dr. Oscar Luis Pyszczek. "LA DIMENSIÓN ESPACIAL DE LA INSEGURIDAD URBANA EN EL SIGLO XXI. PERCEPCIÓN DEL RIESGO Y CONSTRUCCIÓN SOCIAL DEL MIEDO, EN LA CIUDAD DE RESISTENCIA. REPÚBLICA ARGENTINA". RESÚMENES DE TESIS. Revista Geográfica Digital. IGUNNE. Facultad de Humanidades. UNNE. Año 12. № 23. Enero - Junio. 2015. ISSN 1668-5180 Resistencia, Chaco.

En: http://hum.unne.edu.ar/revistas/geoweb/default.htm 
a esta circunstancia y a la vez con intenciones de mostrar su carácter variable, puesto que puede mutar de condiciones si son modificados los factores que la determinan.

\section{Enfoque.}

Es en este marco interpretativo, es dónde se sitúa y emerge el fenómeno que nos convoca. El matiz que la (in)seguridad presenta en su asociación con el delito y el crimen, permanece vinculado a la amenaza, riesgo o temor de los individuos o del colectivo social, ante la posibilidad de sufrir alguna situación que atente contra su integridad física o material. Más allá de su concepción interna, individualmente vivida, y colectivamente compartida, el fenómeno de la (in)seguridad delictiva, procede de componentes objetivos, tales como la frecuencia con que ocurren los hechos delictivos, las reiteradas alusiones por parte de los medios masivos de comunicación, los procedimientos policiales realizados en sitios precisos o el tratamiento riguroso de un caso policial que conmociona a la opinión pública, entre otros. Todo ello proporciona insumos que generan dudas acerca de la eficacia de la protección estatal, en donde la sociedad se percibe y siente vulnerable, o en riesgo. Una vez que se instala en el"consciente o inconsciente" social, la (in)seguridad se dota de un funcionamiento autónomo a las estadísticas delictivas, difícil de revertir.

Las ciencias pioneras dedicadas a la problemática, como la Criminología y la Sociología, han hecho predominar diferentes enfoques en las investigaciones científicas; proporcionando una fuerte impronta en la elaboración del corpus teórico que se proyecta como fuente ineludible de la cuestión.

La geografía como ciencia social, no ha quedado ajena a su abordaje y ha incorporado, como parte de su evolución disciplinar en las últimas décadas del siglo $X X$, a la (in)seguridad delictiva y al crimen, dentro de los campos temáticos emergentes y dentro de los nuevos enfoques teórico-metodológicos. Nuevos problemas y nuevas perspectivas han enriquecido su espectro disciplinar con cuestiones derivadas de la creciente complejidad de la realidad del hombre en este nuevo siglo. El surgimiento de la Geografía de la (in)seguridad ha suscitado creciente interés, particularmente en el estudio del comportamiento espacial del delito en los

Publicado en formato digital: Dr. Oscar Luis Pyszczek. "LA DIMENSIÓN ESPACIAL DE LA INSEGURIDAD URBANA EN EL SIGLO XXI. PERCEPCIÓN DEL RIESGO Y CONSTRUCCIÓN SOCIAL DEL MIEDO, EN LA CIUDAD DE RESISTENCIA. REPÚBLICA ARGENTINA". RESÚMENES DE TESIS. Revista Geográfica Digital. IGUNNE. Facultad de Humanidades. UNNE. Año 12. № 23. Enero - Junio. 2015. ISSN 1668-5180 Resistencia, Chaco.

En: http://hum.unne.edu.ar/revistas/geoweb/default.htm 
centros urbanos-con las respectivas cartografías del crimen- y sus posibilidades de regulación espacial.

Dentro de la Geografía de la (in)seguridad delictiva urbana; la vinculación del espacio con los aspectos perceptivos y vivenciales posibilita el acercamiento a la realidad desde paradigmas que sustentan la importancia de los individuos en la consolidación de la problemática y que a su vez modelan un nuevo tipo de espacio: los espacios del miedo, del peligro.

Las perspectivas que guiaron la investigación adhiere a los postulados de dos corrientes de pensamiento surgidas en la disciplina geográfica en la segunda mitad del siglo XX: la Geografía de la Percepción y la Geografía Humanista, que comparten la concepción del espacio geográfico como un espacio relacional, un producto social que resulta de las prácticas y representaciones de múltiples agentes individuales y colectivos. La combinación de estas dos perspectivas obedece al propósito de lograr una aproximación que en palabras de Hiernaux y Lindón "busca no mutilar la realidad estudiada, sino identificar diversos ángulos para observarla". (2006: 17).

En la actualidad, esta realidad descrita, encuentra en el medio urbano un ámbito casi exclusivo y privilegiado puesto que, en palabras de Curbet “(..) las ciudades, donde la mayoría vivimos gran parte de nuestra vida,(..) son lugares sumamente vulnerables a los trastornos externos" (2008: 180) refiriéndose a las ciudades como elementos indispensables del sistema global.

La existencia y expansión de áreas intraurbanas diferenciadas por sus altos índices de peligrosidad se muestran como "espacios de riesgo y miedo", caracterizando y acompañando la evolución de las capitales y ciudades intermedias de toda América Latina. En este sentido la ciudad de Resistencia, capital provincial con el mayor número de habitantes en el Nordeste y que ha registrado en las últimas décadas una acelerada expansión de su superficie construida, no es la excepción y muestra una significativa segmentación de espacios urbanos relacionados a este fenómeno.El propósito de la tesis es hacer visibles este tipo de espacios invisibles a los ojos de los ciudadanos, pero conocidos implícitamente y vividos cotidianamente por ellos.

Publicado en formato digital: Dr. Oscar Luis Pyszczek. "LA DIMENSIÓN ESPACIAL DE LA INSEGURIDAD URBANA EN EL SIGLO XXI. PERCEPCIÓN DEL RIESGO Y CONSTRUCCIÓN SOCIAL DEL MIEDO, EN LA CIUDAD DE RESISTENCIA. REPÚBLICA ARGENTINA". RESÚMENES DE TESIS. Revista Geográfica Digital. IGUNNE. Facultad de Humanidades. UNNE. Año 12. № 23. Enero - Junio. 2015. ISSN 1668-5180 Resistencia, Chaco.

En: http://hum.unne.edu.ar/revistas/geoweb/default.htm 
En este marco espacial singular, la propuesta de investigación ha pretendido, en modo amplio, desentrañar los aspectos básicos de los espacios del miedo originados por el fenómeno de la (in)seguridad delictiva, e intentar establecer las relaciones entre el fenómeno y los elementos y factores que lo modelan.

El examen a nivel urbano, tiene por objetivo mostrar el contexto formal y funcional, de la dinámica de la seguridad ciudadana provista por los organismos gubernamentales responsables (Policía del Chaco), y la estigmatización y sectorización subjetiva de espacios. Del mismo modo y desde una escala mayor (barrios), se pretendió abordar las consecuencias concretas de las manifestaciones espaciales de la (in)seguridad, la repercusión en la vida cotidiana de los ciudadanos, como también indagar acerca de los procesos de estigmatización de espacios "inseguros" y los factores y elementos convergentes.

La relevancia de la problemática abordada, es manifestada fácticamente en la cotidianeidad y en los diferentes relevamientos sociales realizados por consultoras de alcance nacional y provincial, como por ejemplo el de Poliarquía, realizado en febrero de 2011:

“(...) Consultados sobre los principales problemas que afectan a los argentinos en forma individual, con un $34 \%$ de menciones, la inseguridad encabeza la lista muy lejos del resto. Le siguen el desempleo (15\%) y en tercer lugar la inflación (8\%).

En cambio, cuando se les pregunta por el mayor problema que afecta al país, en la lista se ubica la inseguridad (40\%), el desempleo (11\%), la pobreza (8\%), la educación (7\%), la clase dirigente $(4 \%)$ y recién en el sexto puesto asoma la inflación (3\%), empatada con la corrupción y los problemas económicos en general".3.

$(13 / 02 / 2011)$

La intervención de las políticas de seguridad, enfocadas sobre la base de las percepciones y vivencias cotidianas de los ciudadanos, puede generar paliativos a la sensación de ausencia estatal sobre la cuestión, que hoy en día se arraiga fuertemente sobre la población, y que persiste en el tiempo. La elaboración de respuestas públicas no solo debe orientarse en el sentido objetivo de la cuestión sino involucrar al "termómetro" social.

\footnotetext{
${ }^{3}$ Extraído de http://economia.iprofesional.com/notas/111601-Efecto-K-en-el-ranking-de-preocupaciones-de-losargentinos-la-inflacin-no-ocupa-el-primer-lugar. El término "inseguridad" alude estrictamente a la inseguridad delictiva.
}

Publicado en formato digital: Dr. Oscar Luis Pyszczek. "LA DIMENSIÓN ESPACIAL DE LA INSEGURIDAD URBANA EN EL SIGLO XXI. PERCEPCIÓN DEL RIESGO Y CONSTRUCCIÓN SOCIAL DEL MIEDO, EN LA CIUDAD DE RESISTENCIA. REPÚBLICA ARGENTINA". RESÚMENES DE TESIS. Revista Geográfica Digital. IGUNNE. Facultad de Humanidades. UNNE. Año 12. № 23. Enero - Junio. 2015. ISSN 1668-5180 Resistencia, Chaco.

En: http://hum.unne.edu.ar/revistas/geoweb/default.htm 
El fin es procurar emitir señales de acompañamiento y protección a una sociedad en constante crecimiento, tanto de efectivos humanos como de necesidades y hacer visible los espacios percibidos como (in)seguros, para fomentar el inicio de procesos de deconstrucción de estos estigmas sociales, en pos de mejorar la calidad de vida de los ciudadanos y fomentar la mayor y mejor apropiación tanto material como inmaterial del espacio urbano en Resistencia.

\section{Abordaje.}

La estructura con la que se abordó el objeto de estudio de la tesis, ha implicado una doble perspectiva que suponen aspectos deductivos que surgen del análisis del contexto de la ciudad e inductivos manifestados en la experiencia empírica del trabajo de campo. Los resultados se estructuran en capítulos, -y dentro de ellos,- subtemas y subtítulos que aclaran al conjunto mayor, y que conforman el tomo I (desarrollo teórico) de la Tesis, acompañado del soporte gráfico-cartográfico recopilado en el tomo II.

Los nueve capítulos incluidos en la tesis, están ordenados secuencialmente:

En el primero de ellos, la Delimitación de la problemática, se especifica, delimita y caracteriza el objeto de estudio de la presente investigación, a fin de determinar cabalmente los alcances pretendidos.

Los capítulos correspondientes al Estado del Conocimiento y al Marco teórico, indagan las investigaciones realizadas acerca del objeto de estudio y explicitan el encuadre general que toma la investigación llevada a cabo. El Estado del conocimiento, lejos de ser un producto acabado sobre las investigaciones que aluden a la problemática, tiende a esbozar las líneas investigativas transitadas por las diversas disciplinas de las ciencias sociales, y especialmente por los esfuerzos encarados desde la geografía. En el marco teórico se presentan y especifica las líneas epistemológicas a la que adhiere la investigación como así también, los principales instrumentos conceptuales utilizados.

En el capítulo correspondiente a la metodología, se especifican cada una de las técnicas llevadas a cabo en los diferentes momentos de la investigación, como así también se explican sus características, alcances y limitaciones.

Publicado en formato digital: Dr. Oscar Luis Pyszczek. "LA DIMENSIÓN ESPACIAL DE LA INSEGURIDAD URBANA EN EL SIGLO XXI. PERCEPCIÓN DEL RIESGO Y CONSTRUCCIÓN SOCIAL DEL MIEDO, EN LA CIUDAD DE RESISTENCIA. REPÚBLICA ARGENTINA". RESÚMENES DE TESIS. Revista Geográfica Digital. IGUNNE. Facultad de Humanidades. UNNE. Año 12. № 23. Enero - Junio. 2015. ISSN 1668-5180 Resistencia, Chaco.

En: http://hum.unne.edu.ar/revistas/geoweb/default.htm 
En el capítulo correspondiente al desarrollo, se estructuran los resultados de la investigación, desde el aspecto formal y funcional de la seguridad pública en Resistencia; transitando por la estructura de la (in)seguridad metropolitana desde la visión de sus ciudadanos, hasta el análisis vivencial a escala barrial que supuso la labor realizada con los casos de estudio.

Finalmente el capítulo correspondientes a las conclusiones y Reflexiones finales, subrayan los aportes más importantes de la investigación.

La especificación de las fuentes de información y la bibliografía utilizada, junto con el apartado destinado a los anexos, conforman los dos últimos capítulos de la tesis.

Dos aportes Significativos de la Tesis han sido: A) la sistematización de los aportes geográficos referidos a la Geografía del Crimen e (In)seguridad Delictiva y B) la implementación de una metodología inédita regional.

\section{A) El enfoque Geográfico aplicado a la (In)seguridad delictiva.}

Los trabajos geográficos orientados hacia la (in)seguridad delictiva, variante de la denominada geografía de la (In)seguridad (Cuadro № 1), han atravesado una constante evolución durante todo el siglo pasado. Anteriormente, los principales aportes, referidos a la espacialización de la problemática provinieron del campo de la Sociología y de la Criminalística de fines del siglo XIX y de todo el siglo XX, antecedentes directos de la Geografía del Crimen, cuyas técnicas fueron adoptadas en la mayoría de las investigaciones. Es recién en la segunda mitad del siglo XX dónde se consolida como temática disciplinar dentro de la Geografía, conformando un campo de estudio emergente y promoviendo un serio análisis y debate científico de este acuciante fenómeno social.

Alentados por los fructíferos aportes logrados por Escuela cartográfica de criminología y la Escuela ecológica de Chicago, es en la primera mitad del Siglo XX, (1940 aproximadamente), dónde surgen, desde la Geografía cuantitativa o

Publicado en formato digital: Dr. Oscar Luis Pyszczek. "LA DIMENSIÓN ESPACIAL DE LA INSEGURIDAD URBANA EN EL SIGLO XXI. PERCEPCIÓN DEL RIESGO Y CONSTRUCCIÓN SOCIAL DEL MIEDO, EN LA CIUDAD DE RESISTENCIA. REPÚBLICA ARGENTINA". RESÚMENES DE TESIS. Revista Geográfica Digital. IGUNNE. Facultad de Humanidades. UNNE. Año 12. № 23. Enero - Junio. 2015. ISSN 1668-5180 Resistencia, Chaco.

En: http://hum.unne.edu.ar/revistas/geoweb/default.htm 


\section{Sistematización de la Geografía del Miedo.}

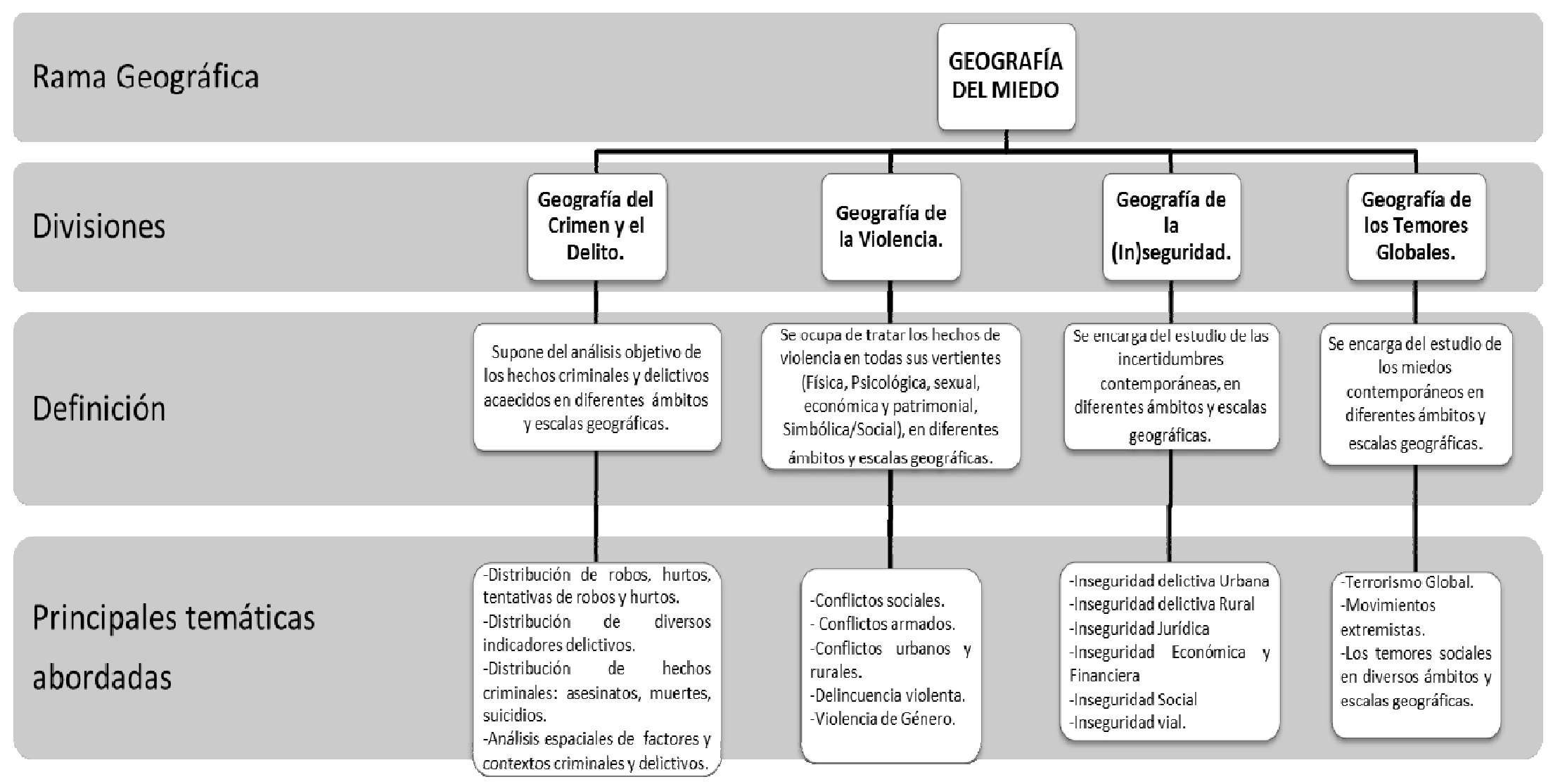

Fuente:Elaboración propia en base a las investigaciones geográficas indagadas, referidas a la (in)seguridad delictiva.

Publicado en formato digital: Dr. Oscar Luis Pyszczek. "LA DIMENSIÓN ESPACIAL DE LA INSEGURIDAD URBANA EN EL SIGLO XXI. PERCEPCIÓN DEL RIESGO Y CONSTRUCCIÓN SOCIAL DEL MIEDO, EN LA CIUDAD DE RESISTENCIA. REPÚBLICA ARGENTINA". RESÚMENES DE TESIS. Revista Geográfica Digital. IGUNNE. Facultad de Humanidades. UNNE. Año 12. № 23. Enero - Junio. 2015. ISSN 1668-5180 Resistencia, Chaco.

En: http://hum.unne.edu.ar/revistas/geoweb/default.htm 
neopositivista, los primeros trabajos geográficos referidos al crimen y a la delincuencia. En ellos se elaboraron mapas referidos a la distribución de robos y hurtos en distintos espacios geográficos como así también otros hechos delictivos, alcanzando relativo éxito (Harries, 1974). Las críticas adjudicadas a este enfoque, no se hicieron esperar y radicaban especialmente, en las falencias atribuidas a las estadísticas oficiales del crimen, las cuales ofrecían una sobrerrepresentación de las clases sociales más bajas y por ende más vulnerables a engrosarlas, siendo excluidos los delitos denominados de "cuello blanco". ${ }^{4}$

Las tendencias reaccionarias al predominio de la geografía positivista la que en los hechos reducía los fenómenos humanos y sus comportamientos a la lógica matemática, y desconocía "la interioridad, subjetividad y sentimientos" de los diversos fenómenos sociales (Santarelli y Campos,2002: 57), produjo que en la década de 1970, la geografía comience a ocuparse críticamente del fenómeno, encausándose en nuevas corrientes epistemológicas disciplinares: la Geografía Radical, de la Percepción y Humanista.

Estos nuevos enfoques cuestionarán a las estadísticas como instrumentos objetivos para arribar a conocimiento válidos, calificándolos de "poco apropiados para conocer la realidad de los delitos y los delincuentes" (Hernando Sanz 2006: 513) y promoverán, entre otros métodos, la utilización de las etnografías, los estudios detallados y la convivencia con el mundo de los desviados para describir su organización y funcionamiento. Cabe resaltar que la evolución alcanzada por la geografía del Crimen y del delito en esta época, supuso un quiebre de tipo técnicometodológico. Más aún, los trabajos geográficos adquirieron una mirada distinta de la misma problemática y en algunos casos resultaron complementarios a las investigaciones realizadas desde el positivismo geográfico, datando de criticidad el campo de estudio, denunciando la estigmatización de las clases populares y planteando líneas de acción alternativas.

\footnotetext{
${ }^{4}$ No obstante, el enfoque liberal reformista, que considera a las estadísticas de delincuencia como indicadores de progreso social y calidad de vida, (muy afín al Nepositivismo geográfico que parecía haber sido superado) adquiere relevancia en las últimas décadas del siglo XX.
}

Publicado en formato digital: Dr. Oscar Luis Pyszczek. "LA DIMENSIÓN ESPACIAL DE LA INSEGURIDAD URBANA EN EL SIGLO XXI. PERCEPCIÓN DEL RIESGO Y CONSTRUCCIÓN SOCIAL DEL MIEDO, EN LA CIUDAD DE RESISTENCIA. REPÚBLICA ARGENTINA". RESÚMENES DE TESIS. Revista Geográfica Digital. IGUNNE. Facultad de Humanidades. UNNE. Año 12. № 23. Enero - Junio. 2015. ISSN 1668-5180 Resistencia, Chaco.

En: http://hum.unne.edu.ar/revistas/geoweb/default.htm 
En la década de los `90, y ante la insuficiencia de la geografía Radical cuestionada por sus afinidad con la teoría Marxista, el estudio del crimen en geografía experimenta una fuerte diversificación temática y metodológica, superando la simple distribución espacial de hechos delictivos, para ocuparse de otros aspectos, tales como los etiquetamientos espaciales, el delito según el género, según grupos etarios, y la relación entre el diseño urbano y el crimen; generando un "estallido" de técnicas, métodos, enfoques y escalas de trabajo, a lo que algunos autores refieren como "eclecticismo metodológico." (Hernando Sanz, 2006.), y se detallan en la Tabla №1.

Tabla № 1. Actuales líneas temáticas del delito y la delincuencia, abordadas desde la Geografía según Sanz, 2006.

\begin{tabular}{|l|l|l|}
\hline LÍNEA TEMÁTICA & PRINCIPALES CONCEPTOS \\
\hline VICTIMIZACIÓN Y MIEDO A LAA & $\begin{array}{l}\text { Estas investigaciones miden el miedo al delito, } \\
\text { interrogando acerca del nivel de seguridad que } \\
\text { tienen las personas entrevistadas en diferentes } \\
\text { circunstancias y cuál es su temor hacia los } \\
\text { diferentes tipos de delincuencia. En estos } \\
\text { trabajos, el miedo al crimen se considera como } \\
\text { un fenómeno social, más que como una faceta } \\
\text { específica de la personalidad de los individuos. }\end{array}$ \\
\hline VIOLENCIA, DELINCUENCIA Y GÉNERO & $\begin{array}{l}\text { A pesar de que los hombres jóvenes son más } \\
\text { proclives a ser víctimas de comportamientos } \\
\text { violentos, el miedo que tienen las mujeres a ser } \\
\text { víctimas de los diferentes tipos de delitos es } \\
\text { mayor, y se manifiesta de forma diferente al de } \\
\text { los hombres. }\end{array}$ \\
\hline $\begin{array}{l}\text { No todas las mujeres comparten la misma } \\
\text { experiencia de miedo en los procesos de } \\
\text { victimización. (Pain, 1997). El miedo de las } \\
\text { mujeres no es "aespacial". Las mujeres }\end{array}$
\end{tabular}

Publicado en formato digital: Dr. Oscar Luis Pyszczek. "LA DIMENSIÓN ESPACIAL DE LA INSEGURIDAD URBANA EN EL SIGLO XXI. PERCEPCIÓN DEL RIESGO Y CONSTRUCCIÓN SOCIAL DEL MIEDO, EN LA CIUDAD DE RESISTENCIA. REPÚBLICA ARGENTINA". RESÚMENES DE TESIS. Revista Geográfica Digital. IGUNNE. Facultad de Humanidades. UNNE. Año 12. № 23. Enero - Junio. 2015. ISSN 1668-5180 Resistencia, Chaco.

En: http://hum.unne.edu.ar/revistas/geoweb/default.htm 


\begin{tabular}{|c|c|}
\hline VIOLENCIA, DELINCUENCIA Y GÉNERO & $\begin{array}{l}\text { muestran un mayor rechazo de los lugares } \\
\text { aislados, por lo que, en muchas ocasiones se } \\
\text { reduce su movilidad y por lo tanto se produce } \\
\text { un diferencial uso del espacio con respecto al } \\
\text { hombre. } \\
\text { Los trabajos realizados sobre la temática } \\
\text { suponen tres vías de aproximación: a) En la } \\
\text { mayor parte de los casos son abordados desde } \\
\text { la perspectiva de teorías ya existentes; b) El } \\
\text { enfoque de la victimización de la mujer desde el } \\
\text { punto de vista de las teorías feministas, c) La } \\
\text { integración de las perspectivas específicas de la } \\
\text { criminología (Rafter y Heidensohn, 1996). }\end{array}$ \\
\hline \multirow[t]{2}{*}{ LOS MEDIOS DELICTIVOS SUBJETIVOS } & $\begin{array}{l}\text { Este tipo de trabajos se preocupan por la trama } \\
\text { subjetiva de los medios locales en donde existe } \\
\text { delincuencia, que en parte facilitan la defensa } \\
\text { de algunas viejas teorías ecológicas. } \\
\text { Los trabajos realizados posibilitan el diagnóstico } \\
\text { no tenidos en cuenta en el resto de } \\
\text { aproximaciones. En los trabajos más recientes se } \\
\text { investigan las dimensiones del dominio afectivo } \\
\text { para caracterizar las principales actitudes y } \\
\text { sentimientos encontrados en las áreas de mayor } \\
\text { criminalidad urbana. Estas dimensiones se } \\
\text { relacionan con diferentes modelos y tratan de } \\
\text { resumir el rango de rasgos que permiten } \\
\text { diferenciar los comportamientos de la } \\
\text { comunidad hacia el crimen y la violencia urbana } \\
\text { en las diferentes áreas residenciales. }\end{array}$ \\
\hline & Desde los momentos iniciales en que Richard \\
\hline
\end{tabular}

Publicado en formato digital: Dr. Oscar Luis Pyszczek. "LA DIMENSIÓN ESPACIAL DE LA INSEGURIDAD URBANA EN EL SIGLO XXI. PERCEPCIÓN DEL RIESGO Y CONSTRUCCIÓN SOCIAL DEL MIEDO, EN LA CIUDAD DE RESISTENCIA. REPÚBLICA ARGENTINA". RESÚMENES DE TESIS. Revista Geográfica Digital. IGUNNE. Facultad de Humanidades. UNNE. Año 12. № 23. Enero - Junio. 2015. ISSN 1668-5180 Resistencia, Chaco.

En: http://hum.unne.edu.ar/revistas/geoweb/default.htm 


\begin{tabular}{|c|c|}
\hline $\begin{array}{l}\text { LA GEOGRAFÍA DE LA DELINCUENCIA, LA } \\
\text { ECONOMÍA POLÍTICA } \\
\text { REVITALIZACIÓN Y DE } \\
\text { ORIENTACIONES MARXISTAS }\end{array}$ & $\begin{array}{l}\text { Peet (1975) propone una lectura crítica de los } \\
\text { trabajos geográficos de violencia urbana, existe } \\
\text { una patente convicción de la necesidad de una } \\
\text { praxis revolucionaria que transforme el estado } \\
\text { de las cosas. Muchos geógrafos manifestaron la } \\
\text { prioridad de combinar con cierta maestría la } \\
\text { teoría y la práctica, pero lo cierto es que en una } \\
\text { gran parte de los casos, eso se quedó en una } \\
\text { mera declaración de intenciones. Dentro de la } \\
\text { orientación debemos destacar algunos trabajos } \\
\text { sobre los aspectos geográficos de la corrupción } \\
\text { política y sus relaciones con la geografía política } \\
\text { (Perry, 1997, Wang, } 1999 \text { y Gallaher, 2004). } \\
\text { En esta orientación marxista de la temática, } \\
\text { existía el convencimiento y la esperanza de } \\
\text { conseguir un cambio social global, de } \\
\text { vislumbrar, en ésta o en una futura generación, } \\
\text { una nueva sociedad al margen del adjetivo que } \\
\text { se le añadiese. }\end{array}$ \\
\hline $\begin{array}{l}\text { EL NARCOTRÁFICO Y EL CONSUMO DE } \\
\text { ESTUPEFACIENTES }\end{array}$ & $\begin{array}{l}\text { En general, el tráfico y consumo de } \\
\text { estupefacientes es una realidad que, aunque } \\
\text { haya contribuido a los vertiginosos incrementos } \\
\text { de la delincuencia y la violencia durante los } \\
\text { últimos treinta años, no ha aparecido en las } \\
\text { agendas de investigación de la geografía } \\
\text { profesional e institucional hasta muy } \\
\text { recientemente. } \\
\text { El consumo y el tráfico de estupefacientes, que } \\
\text { es sin lugar a dudas otro de los problemas } \\
\text { sociales más significativo de las grandes urbes }\end{array}$ \\
\hline
\end{tabular}

Publicado en formato digital: Dr. Oscar Luis Pyszczek. "LA DIMENSIÓN ESPACIAL DE LA INSEGURIDAD URBANA EN EL SIGLO XXI. PERCEPCIÓN DEL RIESGO Y CONSTRUCCIÓN SOCIAL DEL MIEDO, EN LA CIUDAD DE RESISTENCIA. REPÚBLICA ARGENTINA". RESÚMENES DE TESIS. Revista Geográfica Digital. IGUNNE. Facultad de Humanidades. UNNE. Año 12. № 23. Enero - Junio. 2015. ISSN 1668-5180 Resistencia, Chaco.

En: http://hum.unne.edu.ar/revistas/geoweb/default.htm 


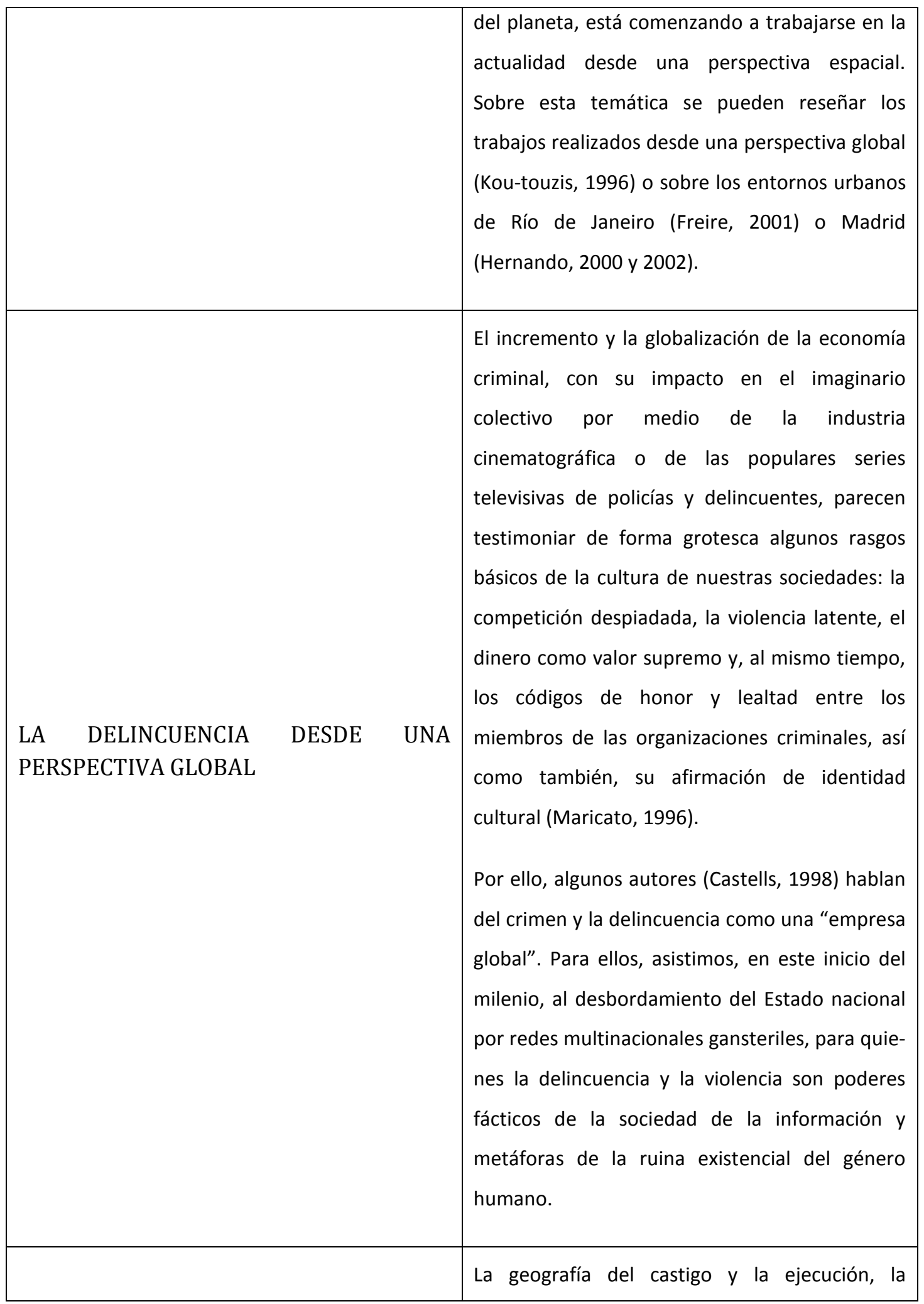

Publicado en formato digital: Dr. Oscar Luis Pyszczek. "LA DIMENSIÓN ESPACIAL DE LA INSEGURIDAD URBANA EN EL SIGLO XXI. PERCEPCIÓN DEL RIESGO Y CONSTRUCCIÓN SOCIAL DEL MIEDO, EN LA CIUDAD DE RESISTENCIA. REPÚBLICA ARGENTINA". RESÚMENES DE TESIS. Revista Geográfica Digital. IGUNNE. Facultad de Humanidades. UNNE. Año 12. № 23. Enero - Junio. 2015. ISSN 1668-5180 Resistencia, Chaco.

En: http://hum.unne.edu.ar/revistas/geoweb/default.htm 


\begin{tabular}{|l|l|l|}
\hline OTROS ÁMBITOS TEMÁTICOS & $\begin{array}{l}\text { seguridad de los espacios y las actividades } \\
\text { EMERGENTES }\end{array}$ \\
turísticas, la prostitución, o las implicaciones \\
territoriales de los ataques terroristas y \\
contraterroristas, son campos actuales de \\
estudio.
\end{tabular}

Fuente: Elaboración propia en base a la sistematización propuesta por Hernando Sanz, 2006.

Asimismo en la última década del siglo $\mathrm{XX}$, comienza a robustecerse los enfoques geográficos de la (in)seguridad, cuyo concepto tiene la propiedad de integrar a la mayoría de las líneas temáticas afines a la geografía del crimen, tales como el miedo, victimización, estigmatización, delitos, etc., de gran interés en la comunidad geográfica. "Dentro del término inseguridad se incluye al miedo, pero también a otros sentimientos que suscitará el delito, como por ejemplo la indignación o la ira, la preocupación política, la expectativa de victimización, así como la serie de acciones individuales y colectivas para hacer frente a las amenazas percibidas." (Bergman y Kessler 2008:211).

Es entonces la Geografía de la (in)seguridad delictiva, la que contiene en parte, a las otras vertientes, y ha evolucionado como una rama que examina de modo integral todos los aspectos de la cuestión, incluyendo, la construcción social de los espacios peligrosos (considerando la dinámica espacial de la inseguridad), la percepción y las vivencias del riesgo y del miedo y las consecuencias de sendos procesos.

Es esta condición de globalidad la que genera gran atracción en los emprendimientos geográficos actuales que investigan el fenómeno, causando en el seno de la Geografía de la (in)seguridad delictiva, la multiplicación de los núcleos de estudios geográficos que tratan, en líneas generales, la misma cuestión, pero se diferencian por los aspectos específicos que se ocupan dentro de la problemática.

Sin fines de exhaustividad se detallan a continuación algunas vertientes de la geografía de la (in)seguridad delictiva en la actualidad:

Publicado en formato digital: Dr. Oscar Luis Pyszczek. "LA DIMENSIÓN ESPACIAL DE LA INSEGURIDAD URBANA EN EL SIGLO XXI. PERCEPCIÓN DEL RIESGO Y CONSTRUCCIÓN SOCIAL DEL MIEDO, EN LA CIUDAD DE RESISTENCIA. REPÚBLICA ARGENTINA". RESÚMENES DE TESIS. Revista Geográfica Digital. IGUNNE. Facultad de Humanidades. UNNE. Año 12. № 23. Enero - Junio. 2015. ISSN 1668-5180 Resistencia, Chaco.

En: http://hum.unne.edu.ar/revistas/geoweb/default.htm 
- La geografía del delito, se encarga de analizar en el espacio, el comportamiento geográfico de los hechos delictivos especificados en la rigurosidad judicial;

- La geografía de la delincuencia, promueve investigaciones destinadas a esclarecer las causas que llevan a los individuos a perpetrar hechos delictivos. Existe una fructífera producción desde los centros de reclusión juvenil y desde las cárceles;

- La geografía de la violencia urbana, trata acerca de los hechos delictivos violentos, sus infractores y actores sociales intervinientes en la ciudad. Cabe resaltar que es una de las líneas de mayor productividad en la actualidad, debido a que la delincuencia se considera un fenómeno exclusivamente urbano adquiriendo relevante presencia.

- La geografía del miedo urbano investiga las manifestaciones espaciales del temor de las personas a ser víctimas de la delincuencia como así también la vinculación con la percepción del riesgo y el peligro. Son sumamente interesantes las encuestas de victimización realizadas fundamentalmente en los países desarrollados y en algunos países emergentes.

- La geografía de los ambientes delictivos, promueve el análisis de los espacios inseguros, peligrosos o de riesgo delictivo, como así también plantea la relación entre el diseño urbano y la posibilidad de delinquir y de ser víctimas de la delincuencia. ${ }^{5}$

B) Sectorización espacial en relación a la (in)seguridad delictiva en Resistencia, según las encuestas de percepción ciudadana.

La situación de (in)seguridad delictiva desde la visión ciudadana, deriva de la implementación de las encuestas sociales (por muestreo) de (in)seguridad delictiva en Resistencia. En pos de optimizar la sistematización de los resultados, se han

\footnotetext{
${ }^{5}$ Esta línea de investigación presenta gran similitud con las estudiadas en la Escuela ecológica de Chicago, especialmente en la obra de Kevin Linch "La imagen de la ciudad" 1959.
}

Publicado en formato digital: Dr. Oscar Luis Pyszczek. "LA DIMENSIÓN ESPACIAL DE LA INSEGURIDAD URBANA EN EL SIGLO XXI. PERCEPCIÓN DEL RIESGO Y CONSTRUCCIÓN SOCIAL DEL MIEDO, EN LA CIUDAD DE RESISTENCIA. REPÚBLICA ARGENTINA". RESÚMENES DE TESIS. Revista Geográfica Digital. IGUNNE. Facultad de Humanidades. UNNE. Año 12. № 23. Enero - Junio. 2015. ISSN 1668-5180 Resistencia, Chaco.

En: http://hum.unne.edu.ar/revistas/geoweb/default.htm 
englobado los ítems consultados, en tres categorías: Identificación de los espacios considerados peligrosos por la (in)seguridad delictiva en la ciudad a escala barrial; factores incidentes en el espacio que lo transforman en (in)seguro desde la óptica ciudadana y las experiencias personales vinculadas a la (in)seguridad delictiva por parte de los encuestados.

En relación a la identificación de los espacios (in)seguros en la ciudad, las consultas realizadas a los encuestados estuvieron representadas por la variable "Nivel de estigmatización de barrios", por la cual se solicitó a los consultados que seleccionaran de la lista de barrios oficiales de la ciudad de Resistencia ${ }^{6}$ aquéllos que consideran delictivamente más (in)seguros.

Previendo casos en los cuales los encuestados desconozcan los topónimos de los barrios, o bien puedan delimitar un sector o sectores que consideren (in)seguros, fue incorporado en la estructura de la encuesta, un plano de la ciudad con los nombres de las diferentes unidades barriales, como así también diferentes puntos de referencia, como cárceles, hospitales, centros educativos, plazas, terminal de ómnibus, avenidas principales entre otras; de modo tal que en la posterior sistematización de los datos, puedan incorporarse esos sectores a las unidades barriales correspondientes.

De la misma manera y ante la posibilidad de que barrios de surgimiento reciente no estén contemplados en la lista, es que se ha brindado la posibilidad de asentar por escrito, el nombre del mismo junto con su localización en la ciudad.

En encuestas "pilotos o de control" realizadas previamente a la consumación oficial, los encuestados-en su mayoría- han seleccionado la totalidad de los barrios de la ciudad como (in)seguros. Éste aspecto de alguna manera anticiparía los resultados que posteriormente fueron confirmados en la implementación oficial de las encuestas, en donde sustancialmente no existen, -según los encuestados- lugares seguros en la ciudad pero sí distintos niveles o grados de (in)seguridad que los diferencia. Para subsanar este aspecto y acotar el universo de los barrios considerados más "peligrosos", es que se solicitó a los encuestados ordenar de modo jerárquico, 5

${ }^{6}$ La lista de barrios utilizada en la encuesta, es la que oficialmente reconocía el Municipio de Resistencia al año 2010.

Publicado en formato digital: Dr. Oscar Luis Pyszczek. "LA DIMENSIÓN ESPACIAL DE LA INSEGURIDAD URBANA EN EL SIGLO XXI. PERCEPCIÓN DEL RIESGO Y CONSTRUCCIÓN SOCIAL DEL MIEDO, EN LA CIUDAD DE RESISTENCIA. REPÚBLICA ARGENTINA". RESÚMENES DE TESIS. Revista Geográfica Digital. IGUNNE. Facultad de Humanidades. UNNE. Año 12. № 23. Enero - Junio. 2015. ISSN 1668-5180 Resistencia, Chaco.

En: http://hum.unne.edu.ar/revistas/geoweb/default.htm 
(cinco) de los barrios identificados previamente, que sobresalgan del resto en cuanto a su peligrosidad.

La concepción, elaboración y aplicación de las encuestas de (in)seguridad delictiva urbana, conforman una experiencia inédita en el ámbito de la ciudad de Resistencia. ${ }^{7}$ Los principales aportes derivados de su consecución pueden ser analizados desde dos puntos de vistas complementarios: el metodológico y el fáctico/cognitivo.

En relación a los aportes metodológicos, se ha logrado corroborar las ventajas de la técnica por sobre otras, en cuanto a la descripción directa de la situación actual del fenómeno en la ciudad. La realización de encuestas supone decidir en cuanto al tamaño muestral, los segmentos de población que integraran la muestra de población (según edad, sexo, nivel socioeconómico, etc.) y los indicadores que reflejaran rasgos sustanciales del fenómeno en cuestión. Los dos primeros ítems, (la determinación del tamaño muestral y los segmentos de población que integrarán la muestra) pueden ajustarse de acuerdo a los diferentes procedimientos científicamente aceptados y a los criterios que el investigador considere adicionar para mejorar o potenciar aspectos de interés, por ejemplo si se buscara sondear la percepción de (in)seguridad por sexo, se dará participación a las ciudadanas excluyendo a masculinos, etc.

Las decisiones referidas a los descriptores o indicadores del fenómeno, supone una reflexión mucho más pormenorizada de los objetivos que se pretendan alcanzar. La concepción y la secuenciación de los indicadores deben evidenciar un equilibrio entre lo pretendido, lo lógico y lo proyectado.

Desde el punto de vista fáctico/cognitivo, las encuestas sobre (in)seguridad delictiva han permitido obtener información que describe la singular visión que los ciudadanos poseen de la situación del fenómeno. Ésta información mediante el análisis de cada uno de los indicadores, ha podido ser transformada en conocimiento sobre la problemática que, incluso, en sus primeras instancias fueron extendidas a los organismos interesados, fundamentalmente la policía del Chaco.

Dentro de los conocimientos alcanzados se destacan:

${ }^{7}$ Por manifestaciones textuales de los funcionarios de la policía del Chaco.

Publicado en formato digital: Dr. Oscar Luis Pyszczek. "LA DIMENSIÓN ESPACIAL DE LA INSEGURIDAD URBANA EN EL SIGLO XXI. PERCEPCIÓN DEL RIESGO Y CONSTRUCCIÓN SOCIAL DEL MIEDO, EN LA CIUDAD DE RESISTENCIA. REPÚBLICA ARGENTINA". RESÚMENES DE TESIS. Revista Geográfica Digital. IGUNNE. Facultad de Humanidades. UNNE. Año 12. № 23. Enero - Junio. 2015. ISSN 1668-5180 Resistencia, Chaco.

En: http://hum.unne.edu.ar/revistas/geoweb/default.htm 
- La sectorización urbana en cuanto a la percepción de in seguridad delictiva.

-La percepción diferencial de los barrios que conforman la ciudad de Resistencia.

- la jerarquización de los barrios de la ciudad de Resistencia en cuanto a su grado o nivel de (in) seguridad.

- Las causas de la percepción de inseguridad diferencial.

- La experiencia ciudadana referida a la inseguridad y al delito.

- La consideración general de la seguridad pública en la ciudad de Resistencia.

Al momento de la sistematización del orden de los barrios más (in)seguros considerados desde el primer lugar al quinto, se observa una fuerte recurrencia de repetición de las mismas unidades barriales. Este hecho denota una asociación muy específica y singular de las mismas, con la (in)seguridad delictiva percibida por los resistencianos. Amén de los datos cuantitativos, esta circunstancia afianzó la certeza de la presencia de unidades barriales que evidencian rasgos específicos de estigmatización espacial.

\section{Referencias Bibliográficas.}

AUGÉ, Marc. Los "no lugares". Espacios del anonimato. Una antropología de la sobre modernidad. Barcelona: Gedisa. 1995.

BAUMAN, Zygmunt. Miedo líquido. La sociedad contemporánea y sus temores. Barcelona: Paidós.2007.

BECK, Ulrich. La sociedad del riesgo: hacia una nueva modernidad. Buenos Aires: Paidós.1998.

DEL CAMPO TEJEDOR, Alberto. Investigar y deconstruir el estigma en barrios marginales. Un estudio de caso. En Zainak. Cuadernos de Antropología-Etnografía, eds. José Ignacio Homobono y Juan Antonio Rubio-Ardanaz, 24: 803-817. Donostia: Euskolkaskuntza. ISBN848419-878-2.2003.

GIDDENS, Anthony. Consecuencias de la modernidad. Madrid: Alianza.1993.

GOFFMAN, Erving. Estigma, la identidad deteriorada. Buenos Aires-Madrid: Amorrortu.1963.

Publicado en formato digital: Dr. Oscar Luis Pyszczek. "LA DIMENSIÓN ESPACIAL DE LA INSEGURIDAD URBANA EN EL SIGLO XXI. PERCEPCIÓN DEL RIESGO Y CONSTRUCCIÓN SOCIAL DEL MIEDO, EN LA CIUDAD DE RESISTENCIA. REPÚBLICA ARGENTINA". RESÚMENES DE TESIS. Revista Geográfica Digital. IGUNNE. Facultad de Humanidades. UNNE. Año 12. № 23. Enero - Junio. 2015. ISSN 1668-5180 Resistencia, Chaco.

En: http://hum.unne.edu.ar/revistas/geoweb/default.htm 
HIERNAUX, Daniel y LINDÓN Alicia. Tratado de Geografía Humana. Barcelona: Anthropos, México: UAMM.2006.

KESSLER, Gabriel. El sentimiento de inseguridad. Sociología del temor al delito. Buenos Aires: Siglo XXI.2006.

LINDÓN, Alicia: "La construcción social del territorio y los modos de vida en la periferia metropolitana." En: Territorios 7: 27-42. Bogotá.2002.

LINDÓN, Alicia: “El constructivismo geográfico y las aproximaciones cualitativas."En: Revista de Geografía Norte Grande37: 5-21. ISSN0718-3402.2007a.

LINDÓN, Alicia. "La ciudad y la vida urbana a través de los imaginarios urbanos". En: Eure (Santiago) 33 (99): 7-16. ISSN0250-7161. 2007b.

LINDÓN, Alicia. "Los imaginarios urbanos y el constructivismo geográfico: los hologramas espaciales." En: Eure (Santiago) 33 (99): 31-46. ISSN0250-7161.2007c.

LÓPEZ, Marcelino; LAVIANA Margarita; FERNÁNDEZ Luis; LÓPEZ Andrés; RODRÍGUEZ Ana María y ALMUDENA Aparicio: "La lucha contra el estigma y la discriminación en salud mental. Una estrategia compleja basada en la in-formación disponible." En: Revista de la Asociación Española de Neuropsiquiatría 28 (101): 43-83. ISSN0211-5735. 2008.

MARTÍN-BARBERO, Jesús. La ciudad: entre medios y miedos. En Ciudadanías del miedo, ed. Susana Rotker, 29-35. Venezuela: Nueva Sociedad.2000.

MAYOL, Pierre. Habitar. En La invención de lo cotidiano. Guadalajara. Universidad Iberoameriana-ITESO.1999.

MUSCAR BENASAYAG, Eduardo F. y FRANCHINI Teresa. "Emplazamientos urbanos en zonas de riesgos naturales: El caso del Gran Resistencia en la planicie chaqueña." En Estudios Geográficos. C.S.I.C. Centro de Investigaciones sobre la Economía, la Sociedad y el Medio (C.I.E.S.M.)53 (208) julio-septiembre: 481-501. Madrid.1992.

NAREDO, María. Seguridad y ciudadanía, necesidad de un pacto de convivencia. Ponencia presentada en la Jornada "Ciudades más seguras", 5 de octubre de 1998. Madrid: Ministerio de Fomento.1998.

ORTEGA VARCÁRCEL, José. La geografía para el siglo XXI. En Geografía humana: procesos, riesgos e incertidumbres en un mundo globalizado, coord. Juan Romero, 25-53. España: Ariel.2004.

RAITER, Alejandro. Representaciones sociales. Buenos Aires: Eudeba. 2002.

REGUILLO CRUZ, Rossana. Losmiedos contemporáneos: sus laberintos, sus monstruos, sus conjuros. En Entre miedos y goces. Comunicación, vida pública y ciudadanías, eds. José Miguel Pereira y Mirla Villadiego. Colombia. Pontificia Universidad Javeriana.2006.

RODRÍGUEZ, Jorge y Camilo ARRIAGADA: "Segregación Residencial en la ciudad latinoamericana." .En Eure (Santiago) 30 (89): 5-24. ISSN0250-7161.2004.

ROTKER, Susana. "Ciudades escritas por la violencia (a modo de introducción)". En: Ciudadanías del miedo, ed. Su-sana Rotker. Caracas: Nueva Sociedad.2000.

Publicado en formato digital: Dr. Oscar Luis Pyszczek. "LA DIMENSIÓN ESPACIAL DE LA INSEGURIDAD URBANA EN EL SIGLO XXI. PERCEPCIÓN DEL RIESGO Y CONSTRUCCIÓN SOCIAL DEL MIEDO, EN LA CIUDAD DE RESISTENCIA. REPÚBLICA ARGENTINA". RESÚMENES DE TESIS. Revista Geográfica Digital. IGUNNE. Facultad de Humanidades. UNNE. Año 12. № 23. Enero - Junio. 2015. ISSN 1668-5180 Resistencia, Chaco.

En: http://hum.unne.edu.ar/revistas/geoweb/default.htm 
SANTARRELLI, Silvia y CAMPOS Marta. Corrientes epistemológicas. Metodología y prácticas en geografía. Propuestas de estudio en el espacio local. Argentina: Departamento de Economía, Universidad Nacional del Sur -Bahía Blanca.2002.

SANZ, Felipe Hernando. "Eclecticismo y diversidad en la geografía del crimen y la delincuencia." En: Anales de Geografía, Universidad Complutense 26: 9-30.2006.

SEGURA, Ramiro. "Paisajes del miedo en la ciudad. Miedo y ciudadanía en el espacio urbano de la ciudad de la Plata". En Cuaderno Urbano. Espacio, Cultura y Sociedad8 (8): 59-91. 2009.

Tuan, Yi-Fu. Topofilia. España: Melusina.1974.

Publicado en formato digital: Dr. Oscar Luis Pyszczek. "LA DIMENSIÓN ESPACIAL DE LA INSEGURIDAD URBANA EN EL SIGLO XXI. PERCEPCIÓN DEL RIESGO Y CONSTRUCCIÓN SOCIAL DEL MIEDO, EN LA CIUDAD DE RESISTENCIA. REPÚBLICA ARGENTINA". RESÚMENES DE TESIS. Revista Geográfica Digital. IGUNNE. Facultad de Humanidades. UNNE. Año 12. № 23. Enero - Junio. 2015. ISSN 1668-5180 Resistencia, Chaco.

En: http://hum.unne.edu.ar/revistas/geoweb/default.htm 